\title{
Lower Bounds for the Disk Packing Constant
}

\author{
By David W. Boyd*
}

\begin{abstract}
An osculatory packing of a disk, $U$, is an infinite sequence of disjoint disks, $\left\{U_{n}\right\}$, contained in $U$, chosen so that, for $n \geqq 2, U_{n}$ has the largest possible radius, $r_{n}$, of all disks fitting in $U \backslash\left(U_{1} \cup \ldots \cup U_{n-1}\right)$. The exponent of the packing, $S$, is the least upper bound of numbers, $t$, such that $\sum r_{n}^{t}=\infty$. Here, we present a number of methods for obtaining lower bounds for $S$, based on obtaining weighted averages of the curvatures of the $U_{n}$. We are able to prove that $S>1.28467$. We use a number of well-known results from the analytic theory of matrices.
\end{abstract}

A packing of a circular disk, $U$, is a sequence of disjoint disks, $U_{1}, U_{2}, \cdots$, contained in $U$, and whose total area equals that of $U$. An osculatory packing is one in which the disks are chosen so that $U_{1}$ is internally tangent to $U$, and $U_{2}, U_{3}, \ldots$ are chosen recursively to have the largest possible radius at each step. If $r_{n}$ denotes the radius of $U_{n}$, then the exponent of the packing, $C=\left\{U_{n}\right\}$, was defined by Melzak [6] to be

$$
S=e(C)=\sup \left\{t: \sum_{n=1}^{\infty} r_{n}^{t}=\infty\right\}
$$

It was shown in [6] that, for osculatory packings, the following bounds hold:

$$
1.035<S<1.999971 \text {. }
$$

In [9], the lower bound was improved to 1.059. Numerical evidence [7] suggests that $S \approx 1.306951$.

Here, we present a method for obtaining lower bounds for $S$. Given any weighted average of the curvatures of the disks in the packing, we obtain such a lower bound. By using a variety of techniques for obtaining these averages, we obtain, successively, the lower bounds, $1.18096,1.25055,1.26154$, and 1.28467. We have no corresponding method, as yet, for upper bounds, but, by using a result of Hirst, we can improve the upper bound in (2) to 1.93113 , and, hence, can replace (2) by

$$
1.28467<S<1.93113 \text {. }
$$

Notation. In this section, we explain a notation used by Hirst [4] for describing the packing of a curvilinear triangle.

Let $T(a, b, c)$ denote the curvilinear triangle formed by three externally tangent circles with curvatures $a, b, c$ where $a \leqq b \leqq c$. We carry out the following procedure: In $T(a, b, c)$, inscribe a circle with curvature, $s$. In the figure so formed, there

Received August 29, 1969.

AMS Subject Classifications. Primary 5245; Secondary 6540.

Key Words and Phrases. Packing of disks, exponent of packing, nonnegative matrix, numerical computation of eigenvalues, Descartes's formula, Soddy's formula, osculatory packing, Apollonian packing.

* This work was supported in part by N.S.F. Grant GP-14133.

Copyright $\odot$ 1971, American Mathematical Society 
are three curvilinear triangles called the first generation of triangles, and, in each of these, we inscribe a circle, calling the three new circles the first generation of circles. Continuing the process in the obvious way, at the $n$th step, we inscribe $3^{n}$ circles (the $n$th generation) in $3^{n}$ curvilinear triangles.

For notation, let $G_{n}=\{1,2,3\}^{n}$, so $G_{n}$ is the set of the $3^{n} n$-tuples with components 1,2 , or 3 . A typical disk in the $n$th generation will be indexed by $\alpha \in G_{n}$. With the disk, $U(\alpha)$, are associated the numbers, $a(\alpha), b(\alpha), c(\alpha)$, which are the curvatures of the triangle, $T(\alpha)$, in which $U(\alpha)$ is inscribed, and the number, $s(\alpha)$, which is the curvature of $U(\alpha)$. Descartes's formula (see [1]) relates $a, b, c$ with $s$ by

$$
s=a+b+c+2(a b+b c+c a)^{1 / 2} .
$$

The other circle tangent to the circles with curvatures $a, b, c$ has curvature $s^{\prime}$ given by

$$
s^{\prime}=a+b+c-2(a b+b c+c a)^{1 / 2} .
$$

In formulas (4) and (5), we must use the convention that, if a circle is externally tangent to the others, its curvature is taken with a negative sign.

We have, thus, the following rules for proceeding from the $n$th to the $(n+1)$ st generation. If $\alpha=\left(i_{1}, \cdots, i_{n}\right)$, write $(\alpha, i)=\left(i_{1}, \cdots, i_{n}, i\right)$. Then

$$
\begin{aligned}
& (a, b, c)(\alpha, 1)=(a, b, s)(\alpha), \\
& (a, b, c)(\alpha, 2)=(a, c, s)(\alpha), \\
& (a, b, c)(\alpha, 3)=(b, c, s)(\alpha) .
\end{aligned}
$$

Equation (6) makes sense, even for $n=0$, if we employ the convention that $G_{0}$ consists of a single element which is a vector with no components. So, for $\alpha \in G_{0}$, we have $a(\alpha)=a, b(\alpha)=b$, etc. We shall write $G=\bigcup_{n=0}^{\infty} G_{n}$.

Now, instead of computing $s(\alpha, i)$ from the formula (4), and the values of $a(\alpha, i)$, $b(\alpha, i), c(\alpha, i)$ in (6), we may compute $s(\alpha, i)$ as follows:

$$
\begin{aligned}
& s(\alpha, 1)=2(a(\alpha)+b(\alpha)+s(\alpha))-c(\alpha), \\
& s(\alpha, 2)=2(a(\alpha)+c(\alpha)+s(\alpha))-b(\alpha), \\
& s(\alpha, 3)=2(b(\alpha)+c(\alpha)+s(\alpha))-a(\alpha) .
\end{aligned}
$$

To verify the first equation in (7), for example, note that the two circles tangent to the circles with curvatures $a(\alpha), b(\alpha)$, and $s(\alpha)$, have curvatures $c(\alpha)$ and $s(\alpha, 1)$, and, hence, formulas (4) and (5) imply (7). (See also [2, p. 111].)

Summarizing, if $g(\alpha)$ is the row vector with components $a(\alpha), b(\alpha), c(\alpha), s(\alpha)$, then

$$
g(\alpha, i)=g(\alpha) P_{i} \quad(i=1,2,3),
$$

where the $P_{i}$ are matrices given by

(9) $P_{1}=\left(\begin{array}{rrrr}1 & 0 & 0 & 2 \\ 0 & 1 & 0 & 2 \\ 0 & 0 & 0 & -1 \\ 0 & 0 & 1 & 2\end{array}\right), \quad P_{2}=\left(\begin{array}{rrrr}1 & 0 & 0 & 2 \\ 0 & 0 & 0 & -1 \\ 0 & 1 & 0 & 2 \\ 0 & 0 & 1 & 2\end{array}\right), \quad P_{3}=\left(\begin{array}{rrrr}0 & 0 & 0 & -1 \\ 1 & 0 & 0 & 2 \\ 0 & 1 & 0 & 2 \\ 0 & 0 & 1 & 2\end{array}\right)$. 
Given $\alpha=\left(i_{1}, \cdots, i_{n}\right) \in G_{n}$, we shall write

$$
P(\alpha)=P_{i_{1}} \cdots P_{i_{n}}
$$

and then $g(\alpha)=g P(\alpha)$.

Note that, by the way the indices are assigned, $a(\alpha) \leqq b(\alpha) \leqq c(\alpha) \leqq s(\alpha)$, for all $\alpha$, and that $s(\alpha, 1) \leqq s(\alpha, 2) \leqq s(\alpha, 3)$, for all $\alpha$. Hence, the smallest curvature in the $n$th generation is $s(1,1, \cdots, 1)$, and the largest is $s(3,3, \cdots, 3)$.

Lower Bounds. In an osculatory packing of a disk $U$ of the type described in the introduction, once $U_{1}, U_{2}$, and $U_{3}$ have been placed, the figure, $U-\left(U_{1} \cup U_{2} \cup U_{3}\right)$, contains a curvilinear triangle, $T(a, b, c)$, with $0<a \leqq b \leqq c$. Hence, as far as obtaining lower bounds for $c$, as defined in (1), we can restrict ourselves to those disks in the packing of $T(a, b, c)$, since the addition of disks to a packing can only raise $S$. Hence, we wish to investigate the set of real numbers, $t$, such that

$$
\sum_{\alpha \in G} s(\alpha)^{-t}=\infty
$$

Suppose that $\{w(\alpha): \alpha \in G\}$ is a set of nonnegative reals which satisfy the following two conditions for some $p$ with $0<p<1$ :

$$
\sum_{\alpha \in G} w(\alpha)^{p}=\infty \text { and } \sum_{\alpha \in G} w(\alpha) s(\alpha)<\infty .
$$

Then, by Hölder's inequality [3, p. 24], we have

$$
\left\{\sum_{\alpha \in G} w(\alpha)^{p}\right\}^{1 / p} \leqq\left\{\sum_{\alpha \in G} w(\alpha) s(\alpha)\right\} \cdot\left\{\sum_{\alpha \in G} s(\alpha)^{-\imath}\right\}^{1 / t},
$$

where $t=p /(1-p)$. Hence, (12) and (13) imply (11).

The remainder of the paper is devoted to obtaining weights, $w(\alpha)$, for which the conditions of (12) hold. The theoretical results are contained in Theorems 1 and 2, and the applications of these to obtain explicit lower bounds follow the respective theorems. We shall use a number of well-known results from the analytic theory of matrices for which we refer the reader to [5] and [8].

TheOrem 1. Let $P_{1}, P_{2}, P_{3}$, and $P(\alpha)$ be defined as in (9) and (10). Let $m$ be a positive integer and let $x(\alpha)\left(\alpha \in G_{m}\right)$ be any $3^{m}$ positive real numbers. Let

$$
A=\sum_{\alpha \in G_{m}} x(\alpha) P(\alpha)
$$

Then the matrix $A$ has a positive eigenvalue $\rho$ which strictly dominates all other eigenvalues and is a simple root of the characteristic equation of $A$. The following equation has a solution $p$ with $0<p<1$

$$
\sum_{\alpha \in G_{m}} x(\alpha)^{p}=\rho^{p}
$$

and if $t=p /(1-p)$, then $t \leqq S$, where $S$ is defined by (1).

Proof. Let $Q$ be the matrix with zeros below the main diagonal and ones above and on the main diagonal. For $i=1,2,3$, let $R_{i}=Q P_{i} Q^{-1}$. Then

$$
R_{1}=\left(\begin{array}{llll}
1 & 0 & 0 & 4 \\
0 & 1 & 0 & 2 \\
0 & 0 & 1 & 0 \\
0 & 0 & 1 & 1
\end{array}\right), \quad R_{2}=\left(\begin{array}{llll}
1 & 0 & 0 & 4 \\
0 & 1 & 0 & 2 \\
0 & 1 & 0 & 3 \\
0 & 0 & 1 & 1
\end{array}\right), \quad R_{3}=\left(\begin{array}{llll}
1 & 0 & 0 & 4 \\
1 & 0 & 0 & 5 \\
0 & 1 & 0 & 3 \\
0 & 0 & 1 & 1
\end{array}\right) .
$$


Thus, $R_{i}$ is nonnegative for each $i$, and $R_{3}$ is irreducible [5, p. 48]. Hence, $A$ is similar to a nonnegative irreducible matrix and, thus, by the Perron-Frobenius theorem [5, p. 49], has its spectral radius $\rho$ as a simple eigenvalue. Furthermore, $R_{3}^{\prime}>0$ for $\nu \geqq 3$, so $R_{3}$ is primitive and, hence, $Q A Q^{-1}$ is primitive [5, p. 59, Exercises 17 and 21]. Thus, $\rho$ is strictly greater in absolute value than all other eigenvalues.

Next, note that if $h$ is the column vector with all components equal to 1 , then, for $i=1,2,3, R_{i} h \geqq h$, with strict inequality, if $i=2$ or 3 . Hence, $R_{i_{1}} \cdots R_{i_{m}} h>h$, unless $i_{1}=i_{2}=\cdots=i_{m}=1$. Using this fact, we see that $Q A Q^{-1}$ has row sums all strictly greater than $\sum_{\alpha \in \sigma_{m}} x(\alpha)$, so that $\rho>\sum_{\alpha \in \sigma_{m}} x(\alpha)$ [5, p. 49].

To show that (14) has a solution $p$ with $0<p<1$, consider the following function:

$$
f(p)=\sum_{\alpha \in G_{m}}(x(\alpha) / \rho)^{p} .
$$

The function $f$ is strictly decreasing and continuous. Also, $f(0)=3^{m}$, and $f(1)<1$ by the above paragraph, so the existence of $p$ satisfying $f(p)=1$ follows.

Finally, we show that $S \geqq p /(1-p)$. Given any $\epsilon>0$, let $T=(\rho+\epsilon)^{-1} A$. Then $T$ has spectral radius $\rho(\rho+\epsilon)^{-1}<1$, so the series $\sum_{n=0}^{\infty} T^{n}$ converges [5, p. 54].

We examine the individual terms $T^{n}$ in this series. Let $\xi(\alpha)=x(\alpha) \cdot(\rho+\epsilon)^{-1}$, and we see that

$$
T^{n}=\left(\sum_{\alpha \in G_{m}} \xi(\alpha) P(\alpha)\right)^{n}=\sum_{\beta \in G_{n m}} w(\beta) P(\beta),
$$

where for $\beta \in G_{n m}$, if $\beta=\left(i_{11}, \cdots, i_{1 m}, i_{21}, \cdots, i_{2 m}, \cdots, i_{n 1}, \cdots, i_{n m}\right)$, write $\alpha_{k}=$ $\left(i_{k 1}, \cdots, i_{k m}\right)$, and then $w(\beta)=\xi\left(\alpha_{1}\right) \cdots \xi\left(\alpha_{n}\right)$. In general, if $\gamma \in G_{n m+k}$ with $0 \leqq$ $k \leqq m-1$, write $\gamma=\left(i_{11}, \cdots, i_{1 m}, \cdots, i_{n 1}, \cdots, i_{n m}, j_{1}, \cdots, j_{k}\right)$, and $\alpha_{k}=\left(i_{k 1}, \cdots, i_{k m}\right)$. Then, define

$$
w(\gamma)=\xi\left(\alpha_{1}\right) \cdots \xi\left(\alpha_{n}\right)
$$

and we have

$$
\left(\sum_{n=0}^{\infty} T^{n}\right)\left(\sum_{k=0}^{m-1} \sum_{\alpha \in G_{k}} P(\alpha)\right)=\sum_{n=1}^{\infty} \sum_{k=0}^{m-1} \sum_{\beta \in G_{m}} \sum_{\alpha \in G_{k}} w(\beta) P(\beta) P(\alpha)=\sum_{\gamma \in \sigma} w(\gamma) P(\gamma) .
$$

Hence, since $\sum_{n=1}^{\infty} T^{n}$ converges, we see from (17) that $\sum_{\gamma \in G} w(\gamma) P(\gamma)$ converges, and a fortiori that $\sum_{\gamma \in \theta} w(\gamma) s(\gamma)$ converges.

We can obtain an expression for $\sum_{\gamma \in G} w(\gamma)^{p}$ by noting that $w(\gamma)$ is defined multiplicatively in terms of the $\xi(\alpha)$.

Hence, replacing $\xi(\alpha)$ by $\xi(\alpha)^{p}$, and retracing the derivation of (17) with $P_{1}, P_{2}, P_{3}$ replaced by the number one, we see that

$$
\sum_{n=0}^{\infty}\left(\sum_{\alpha \in G_{m}} \xi(\alpha)^{p}\right)^{n} \cdot\left(\sum_{k=0}^{m-1} 3^{k}\right)=\sum_{\gamma \in G} w(\gamma)^{p} .
$$

Thus, if $p=p(\epsilon)$ is chosen so that

$$
\sum_{\alpha \in G_{m}} \xi(\alpha)^{p(\epsilon)}=1
$$

then $\sum w(\gamma)^{p(\epsilon)}$ diverges, and, hence, by our earlier remarks, $\sum s(\gamma)^{-t(\epsilon)}$ diverges, where $t(\epsilon)=p(\epsilon) /(1-p(\epsilon))$. But $p(\epsilon)$, as defined by (19), is a strictly increasing function with a strictly increasing inverse for $\epsilon$ in some neighbourhood of $\epsilon=0$. Thus, 
$p(\epsilon)$ is continuous at 0 , and so, $S \geqq t(\epsilon)$ for $\epsilon>0$ implies $S \geqq t(0)=p(0) /(1-p(0))$, which is what is what we wanted to prove.

Explicit Bounds. 1. First, take $m=x(1)=x(2)=x(3)=1$ in Theorem 1. Then the eigenvalues of $A=P_{1}+P_{2}+P_{3}$ may be explicitly calculated as $0,1,4-(13)^{1 / 2}$, $4+(13)^{1 / 2}$, respectively, so $\rho=4+(13)^{1 / 2}$. The equation for $p$ reduces to $3=$ $\left(4+(13)^{1 / 2}\right)^{p}$, so we obtain

$$
S \geqq \log 3 / \log \left(\left(4+(13)^{1 / 2}\right) / 3\right)=1.18096 \cdots .
$$

We can think of this use of Theorem 1 as estimating $\sum s(\alpha)^{-t}$ by replacing $s(\alpha)$ for $\alpha \in G_{n}$ by

$$
3^{-n} \sum_{\alpha \in G_{n}} s(\alpha) \sim\left(\frac{4+(13)^{1 / 2}}{3}\right)^{n} .
$$

(The legitimacy of this replacement follows from (13).) This can be compared with Melzak's lower bound 1.035, which is obtained by replacing $s(\alpha)$ for $\alpha \in G_{n}$ by the maximum curvature in $G_{n}$, which is $s(3,3, \cdots, 3)$.

2. A better choice for $x(1), x(2), x(3)$, with $m=1$, can be heuristically justified by noting that the dominant eigenvalues of $P_{1}, P_{2}, P_{3}$ are $1, \tau^{2}=2.6180 \cdots$, and $\tau+\sqrt{ } \tau=2.89005 \cdots$, respectively. (Here, $\tau=(\sqrt{ } 5+1) / 2$.) Thus, it would seem natural to weight $P_{1}, P_{2}, P_{3}$, roughly in the proportions $1: \tau^{-2}: \tau-\sqrt{ } \tau$. We resorted, at this point, to numerical techniques. One notes that $A$ is similar to a nonnegative irreducible matrix, so that if $g$ is any vector of the form $h Q$ with $h_{i}>0$, (i.e. $g_{1}<g_{2}<g_{3}<g_{4}$ ), and, if we define $g^{(\nu)}=g^{(\nu-1)} A$, and

$$
\beta_{\nu}=\max _{1 \leq i \leq 4}\left(g_{i}^{(v)} / g_{i}^{(v-1)}\right), \quad \alpha_{\nu}=\min _{1 \leq i \leq 4}\left(g_{i}^{(v)} / g_{i}^{(v-1)}\right),
$$

then $\alpha_{1} \leqq \alpha_{2} \leqq \cdots \leqq \alpha_{v} \leqq \cdots \leqq \rho \leqq \cdots \leqq \beta_{\nu} \leqq \cdots \leqq \beta_{1}$ (see [5, p. 60]), and $\beta_{n}, \alpha_{n} \rightarrow \rho$, since $\rho$ strictly dominates the other eigenvalues of $A$.

Thus, improved upper and lower bounds can be obtained for $\rho$ by simple iteration, and these will converge to $\rho$. We used this procedure for a number of choices of $x, y, z$, starting from the above guess, determining $p$ as the unique solution of $x^{\nu}+y^{p}+$ $z^{\nu}=\rho^{p}$ by the method of regula falsi. We found that the choice $(x(1), x(2), x(3))=$ $(.63, .21, .16)$ gave $\rho=2.213092$ and $t=1.250556$, so that $S \geqq 1.25055$.

3. When $m=2$, we are dealing with 9 weights. However, we can immediately do as well as in our last example by taking $x(i, j)=x(i), x(j)$, where $x(i), i=1,2,3$, are $.63, .21, .16$, respectively. Starting with this choice, we eventually settled on the values:

$(x(1,1), x(1,2), x(1,3), \cdots, x(3,3))=(1.10, .17, .15, .17, .09, .08, .15, .06, .05)$,

which gave $\rho=8.9443431$ and $t=1.2615451$, so now $S \geqq 1.26154$.

An Improved Method. The following method, which apparently gives better bounds than that of Theorem 1, was motivated by considering the defects of the weighting scheme in Theorem 1 . We observe that $P_{1}$ has all eigenvalues equal to 1 , and its canonical form has one block of size $3 \times 3$, the other of size $1 \times 1$. Hence, a typical vector of the form $g P_{1}^{n}$ grows like $n^{2}$. On the other hand, $P_{2}$ and $P_{3}$ have simple strictly dominant eigenvalues so typical vectors $g P_{2}^{n}, g P_{3}^{n}$ grow geometrically like $\tau^{2 n},(\tau+\sqrt{ } \tau)^{n}$. The weighting scheme of Theorem 1, however, weights higher 
powers of $P_{1}$ as if they grew geometrically, and so the weights $w\left(i_{1}, i_{2}, \cdots, i_{n}\right)$ are unlike the ideal weights, $s\left(i_{1}, i_{2}, \cdots, i_{n}\right)^{-t-1}$, which would give equality in (13).

Theorem 2 uses a method of weighting which can partially remedy this defect.

THEOREM 2. Let $\{x(n): n=1,2, \cdots\}$ be a sequence of nonnegative reals for which the following three series converge:

$$
f=\sum_{n=1}^{\infty} x(n), \quad f^{\prime}=\sum_{n=1}^{\infty} n \cdot x(n), \quad f^{\prime \prime}=\sum_{n=1}^{\infty} n(n-1) \cdot x(n) .
$$

Let $F$ be the following matrix:

$$
F=\left(\begin{array}{cccc}
f & 0 & 2 f^{\prime \prime} & 4 f^{\prime} \\
0 & f & f^{\prime \prime} & 2 f^{\prime} \\
0 & 0 & f & 0 \\
0 & 0 & f^{\prime} & f
\end{array}\right) .
$$

Let $y$ and $z$ be positive reals, and let $T$ be the matrix $T=y R_{2}+z R_{3}$, where $R_{2}$ and $R_{3}$ are given in (15).

Then, the matrix $M=T(I+F)$ has a strictly dominant eigenvalue $\rho>0$. The following equation has a solution $p$ with $0<p<1$

$$
\left(y^{p}+z^{p}\right)\left(1+\sum_{n=1}^{\infty} x(n)^{p}\right)=\rho^{p},
$$

and, if $t=p /(1-p)$, then $S \geqq t$, where $S$ is given in (1).

Proof. The matrix $F$ of (22) is precisely the matrix $\sum_{n=1}^{\infty} x(n) R_{1}^{n}$, where $R_{1}$ is given in (15). This can be seen by reduction to canonical form or directly, first establishing the form of $R_{1}^{n}$ by induction. The conditions that the series $f, f^{\prime}, f^{\prime \prime}$ converge ensure that the series $\sum x(n) R_{1}^{n}$ converges.

Thus, the matrix $M$ is

$$
M=\left(y R_{2}+z R_{3}\right) \cdot\left(I+\sum_{n=0}^{\infty} x(n) R_{1}^{n}\right) .
$$

The matrix $M$ is nonnegative (obviously), irreducible, since $M \geqq z R_{3}$, and primitive for the same reason. Thus, $M$ has a strictly dominant eigenvalue $\rho$. Evaluating row sums as in Theorem 1, we see that, if $h=(1,1,1,1)^{T}$, then $(I+F) h \geqq(1+f) h$, and so $M h \geqq\left(y R_{2}+z R_{3}\right)(1+f) h \geqq 2(y+z)(1+f) h$, so that $\rho>2(y+z)(1+f)$. The same argument as in Theorem 1 now shows that Eq. (23) has a solution $p$ with $0<p<1$.

Now, given $\epsilon>0$, let $N=(\rho+\epsilon)^{-1} M$, so that the series $\sum_{n=0}^{\infty} N^{n}$ converges, and, equally, the series $\sum_{n=0}^{\infty}\left(Q^{-1} N Q\right)^{n}$ converges, where $Q$ is as in the proof of Theorem 1. If $\eta=y(\rho+\epsilon)^{-1}, \zeta=z(\rho+\epsilon)^{-1}$, we have

$$
Q^{-1} N Q=\left(\eta P_{2}+\zeta P_{3}\right)\left(I+\sum_{n=1}^{\infty} x(n) P_{1}^{n}\right) \text {. }
$$

Thus,

$$
\left(Q^{-1} N Q\right)^{n}=\sum_{\alpha \in H_{n}} w(\alpha) P(\alpha),
$$

where $H_{n}^{\prime}$ consists of vectors $\left(i_{1}, \cdots, i_{r}\right)$, with $r$ arbitrary, but with exactly $n$ com- 
ponents chosen from the set $\{2,3\}$, and with $i_{1} \in\{2,3\}$. (The weights $w(\alpha)$ will be discussed later.)

If we now let $H_{n}$ consist of vectors $\left(i_{1}, \cdots, i_{r}\right)$, with exactly $n$ components chosen from $\{2,3\}$, without the restriction $i_{1} \in\{2,3\}$, then

$$
\begin{aligned}
\sum_{\alpha \in H_{n}} w(\alpha) P(\alpha) & =\left(I+\sum_{k=1}^{\infty} x(k) P_{1}^{k}\right)\left(Q^{-1} N Q\right)^{n} \\
& =\left(I+Q^{-1} F Q\right)\left(Q^{-1} N Q\right)^{n} .
\end{aligned}
$$

Clearly, $G=\bigcup_{n=0}^{\infty} H_{n}$, where the union is a disjoint union, and, hence, from (27) we obtain

$$
\sum_{\alpha \in Q} w(\alpha) P(\alpha)=\left(I+Q^{-1} F Q\right) \sum_{n=0}^{\infty}\left(Q^{-1} N Q\right)^{n} .
$$

This proves that $\sum w(\alpha) P(\alpha)$, and, hence, $\sum w(\alpha) s(\alpha)$ converges.

The weights $w(\alpha)$ are defined through the Eqs. (25), (26), (27), and can be seen to be products of various $x(k)$ 's, $\eta$ and $\zeta$. More precisely, to obtain $w(\alpha)$ from $\alpha$, we replace each block of $k$ consecutive 1's by $x(k)$ (where $k$ is chosen maximally), then replace each 2 by $\eta$, and each 3 by $\zeta$, and compute the product of these numbers.

As in the proof of Theorem 1, we may obtain $\sum w(\alpha)^{p}$ by replacing each $P_{i}$ in (25) by the number 1 , and $\eta, \zeta, x(n)$ by $\eta^{p}, \zeta^{p}, x(n)^{p}$, and substituting in (28) to obtain

$$
\sum_{\alpha \in G} w(\alpha)^{p}=\sum_{n=0}^{\infty}\left(1+\sum_{k=1}^{\infty} x(k)^{p}\right)^{n+1}\left(\eta^{p}+\zeta^{p}\right)^{n} .
$$

The series in (29) diverges if $p=p(\epsilon)$ is chosen to satisfy

$$
\left\{1+\sum_{k=1}^{\infty} x(k)^{p}\right\}\left(y^{p}+z^{p}\right)=(\rho+\epsilon)^{p} .
$$

Again, $p$ is strictly increasing with $\epsilon$ and has a strictly increasing inverse in a neighborhood of $\epsilon=0$, so that $p$ is continuous in $\epsilon$. From the divergence of (29) and the convergence of (28), we have $S \geqq p(\epsilon) /(1-p(\epsilon))$, for $\epsilon>0$, and, by continuity of $p$, we have $S \geqq p(0) /(1-p(0))=t$.

Explicit Bounds. 4. For our first application of Theorem 2, we chose $x(n)=x \cdot n^{-\bullet}$, where $x$ and $s$ are real parameters. Because of homogeneity in $y$ and $z$, it sufficed to take $y+z=1$. The series (21) were, then, $f=x \zeta(s), f^{\prime}=x \zeta(s-1), f^{\prime \prime}=$ $x(\zeta(s-2)-\zeta(s-1))$. Starting with the guess $x=2, s=4.6, y=z=.5$, we systematically improved the lower bound until for $x=.25, s=3.4, y=.55, z=.45$, we obtained the eigenvalue 4.968321 , and the lower bound $S \geqq 1.26671$. The zeta function was computed using nine terms of the series and four terms of the EulerMaclaurin remainder.

5. Next, we chose $x(n)=x \cdot n^{-s}$, for $n=P, P+1, \cdots, Q$, and $x(n)=0$ otherwise. This led to a considerable improvement of the above and the choice, $x=.18, s=2.7, y=.55, z=.45, P=1, Q=20$, gave the eigenvalue 4.944790 and the lower bound $S \geqq 1.28467$. It, perhaps, should be noted here that we have discarded all those $\alpha$ which have more than 20 consecutive components equal to 1 .

Upper Bounds. As we mentioned earlier, we have not yet been able to obtain good upper bounds on $S$ by a similar technique. We should mention an improvement of (2) which can be obtained from a result of Hirst [4]. Let $r(\alpha)$ denote the radius of the 
circumscribed circle to the triangle $T(a(\alpha), b(\alpha), c(\alpha))$. Then, obviously, we have $r(\alpha)>s(\alpha)^{-1}$. The following inequality is due to Hirst [4, p. 285]:

$$
\sum_{\alpha \in G_{n}} r(\alpha)^{x} \leqq(a b+b c+c a)^{-x / 2},
$$

where $x=\log 3 / \log \left(1+\frac{2}{3} \sqrt{ } 3\right)=1.43113 \cdots$.

Denoting the constant in the right member of (30) by $K$, we thus have

$$
\sum_{\alpha \in G_{n}} s(\alpha)^{-x}<\sum_{\alpha \in G_{n}} r(\alpha)^{x} \leqq K .
$$

We know that for $\alpha \in G_{n}, s(\alpha) \geqq s(1, \cdots, 1)=t_{n}$, say. By Lemma 4 of [6], $t_{n}=$ $(a+b)(n+1)^{2}+2(n+1)[a c+b c+a b]^{1 / 2}+c$, so that $s(\alpha)>(a+b)(n+1)^{2}$. Hence, if $\delta>1 / 2$,

$$
\sum_{\alpha \in G_{n}} s(\alpha)^{-x-\delta} \leqq(a+b)^{-\delta}(n+1)^{-2 \delta} \sum_{\alpha \in G_{n}} s(\alpha)^{-x} \leqq K^{\prime}(n+1)^{-2 \delta}
$$

and, thus, $\sum_{\alpha \in G} s(\alpha)^{-x-\delta} \leqq K^{\prime} \sum_{n=0}^{\infty}(n+1)^{-2 \delta}$ converges. Since this holds for any $\delta>1 / 2$, we have $S \leqq x+.5=1.93113 \cdots$.

Final Remarks. We should perhaps point out that having the matrices similar to nonnegative matrices is not entirely essential to the success of the method described here, since, in Theorems 1 and 2, we could simply replace $\rho$ by $|\rho|$ if the dominant eigenvalue were not positive. It is rather useful, however, to know that the spectral radius is a strictly dominant eigenvalue, since then we can obtain $\rho$ to arbitrary accuracy by simple iteration.

It seems quite certain that better results can be obtained from Theorem 2 by a different choice of the sequence $\{x(n)\}$.

All the computations involved were performed on the California Institute of Technology time-sharing system, using the Citran language. This on-line system was well adapted to our problem, since it allowed a heuristic search for large lower bounds from each method without employing unnecessarily sophisticated techniques.

California Institute of Technology

Pasadena, California 91109

1. H. S. M. Coxeter, "The Problem of Apollonius," Amer. Math. Monthly, v. 75, 1968, pp. 5-15. MR 37 \#5767.

2. H. S. M. CoXeter, "Loxodromic sequences of tangent spheres," Aequationes Math., v. 1, 1968, pp. 104-121. MR 38 \#765. York, 1934 .

年, Pólya, Inequalities, Cambridge Univ. Press, New

4. K. E. HIRst, "The Apollonian packing of circles," J. London Math. Soc., v. 42, 1967, pp. 281-291. MR 35 \#876.

5. A. S. Householder, The Theory of Matrices in Numerical Analysis, Blaisdell, Waltham, Mass., 1964. MR 30 \#5475.

6. Z. A. MelzAK, "Infinite packings of disks," Canad. J. Math., v. 18, 1966, pp. 838-852. MR 34 \#3443.

7. Z. A. MelzaK, "On the solid-packing constant for circles," Math. Comp., v. 23, 1969, pp. 169-172.

8. H. Wieland, Topics in the Analytic Theory of Matrices, Lecture Notes, University of Wisconsin, 1967.

9. J. B. Wilker, “Open disk packings of a disk," Canad. Math. Bull., v. 10, 1967, pp. 395415. MR 35 \#6041. 